\title{
Playout Buffer Aware Adaptation Scheme over Multi-client LTE networks
}

\author{
Yuchen Chen \\ School of Electronic and \\ Information Engineering \\ Xi'an Jiaotong University \\ Xi'an, China \\ chen.ych@stu.xjtu.edu.cn
}

\author{
Guizhong Liu \\ School of Electronic and \\ Information Engineering \\ Xi'an Jiaotong University \\ Xi'an, China \\ liugz@mail.xjtu.edu.cn
}

\author{
Qinli Wang \\ School of Electronic and \\ Information Engineering \\ Xi'an Jiaotong University \\ Xi'an, China \\ w.00152651@stu.xjtu.edu.cn
}

\begin{abstract}
Nowadays, HTTP adaptive streaming (HAS) has become a cost-effective solution in delivering the video content. Different from the one-client HAS, the HAS over multi-client faces many challenges. Due to the lack of the knowledge of the networks, one-client HAS will leads to the low efficiency in utilizing the limited bandwidth over the multi-client circumstance. In this paper, a playout buffer aware adaptation scheme over multi-client (PBMC) LTE networks is proposed to improve the performance of HAS. Firstly, a QoE metric is given that considers both the static quality and the quality smoothness. Secondly, to maximize the QoE, an integer programming problem is formulated. By considering the playout buffer level, the resource requirement, which can guarantee the playout continuity, is formulated as a constraint of the problem. Then, the feasibility of the problem is analyzed. If the problem is infeasible, a method is given to exclude the users as less as possible to obtain the feasibility. When the problem is feasible, a graph model can be constructed based on the resource requirement and the playout buffer level. With the model, the solution can be converted to finding a feasible path with the highest QoE in the graph, which can be solved by employing the dynamic programming. Finally, the simulation results can verify the performance of PBMC in both improving the static quality and guaranteeing the playout continuity.
\end{abstract}

\section{Categories and Subject Descriptors}

H.4 [Information Systems Applications]: Miscellaneous

\section{General Terms}

Algorithms,Performance

\section{Keywords}

Playout Buffer, Adaptation, streaming, LTE, Multi-client

\section{INTRODUCTION}

With the development of the wireless networks and the mobile equipments, video streaming has increased its popularity dramatically in the recent years. According to the report from Cisco, the mobile video will grow faster than any other mobile service with the CAGR (Compound Average Growth Rate) of 69 percent from 2013 to 2018[4]. Thanks to the flexibility of the Web platform, HTTP adaptive streaming (HAS) has become a cost-effective solution in delivering video content. The source video sequences are pre-encoded into several versions with different quality level. Meanwhile, a video sequences will be split into small segments, each of which contains several video frames. The clients dynamically request the video segments at each switching point based on its estimated network condition and its playout status.

The basic objective of HAS is to sustain the continuous playout process. In [2], the transmission capability of the network is estimated used to decided which version to request. In [10], a more complicated estimation method is proposed, which considers both the past and instant throughput. In fact, not only the throughput but also the playout buffer status should be taken into consideration when making adaptation decisions. In [9], the buffer level is split into several ranges. According to the range that the buffer level lies in, different actions will be applied to keep the buffer level steady. For instance, if the buffer level is low, the UE would request for the version with low quality to avoid the playout interruption. On the contrary, when the buffer level is high, the higher version would be requested to improve the static quality and the quality smoothness. Although the schemes described above can bring some improvements of the performance, experimental results showed that the competition of the shared network resource in the bottleneck could lead to degradation in fairness, stability and efficiency, which would negatively impact the QoE of the HAS users[1][8]. In [3], the disadvantage of the client-side adaptation is introduced specifically, which can be described as follows. Because of lacking the knowledge of other UEs, the client-side solutions cannot control the UEs to share the resource in a coordinative way, which would results in incorrect throughput estimations, unnecessary quality oscillations and suboptimal decisions. The possibility of locating the adaptation in network has been shown in this paper. In [12], the adaptation problem is formulated as an optimization problem, which jointly determine the requests of all the UEs together. However, the playout continuity cannot be guaranteed by the model, which is an important QoE factor. This would bring 


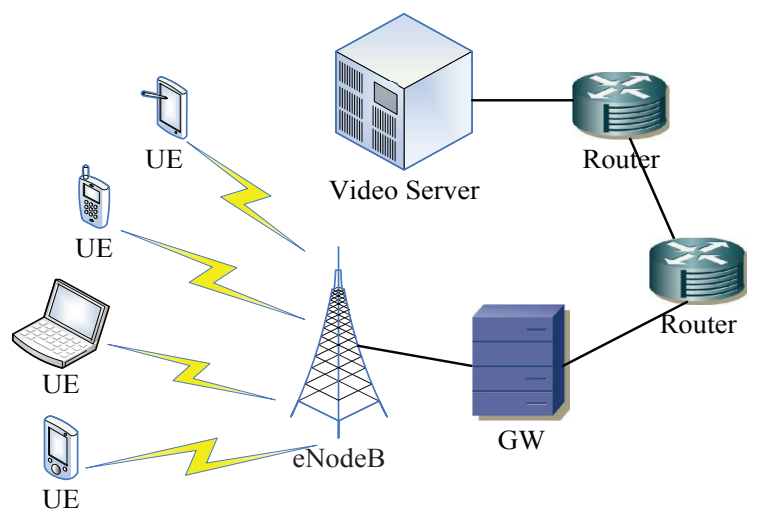

Figure 1: The Topology of the Network

a severe limitation of its performance in improving the HAS performance over multi-client circumstance.

To improve the performance of HAS, the playout buffer aware adaptation scheme over multi-client LTE networks is proposed in this paper, which has the novel aspects as follows:

1) QoE assessing metric. By analyzing the weaknesses of the existing assessing schemes, an novel QoE assessing metric is given, which considers the static quality and the quality variation, where the quality variation is considered as a modification of the static quality.

2) Adaptation problem formulation. The adaptation problem is formulated as an integer programming problem, aiming at maximizing the total QoE of all the users. By considering the occupancy of the playout buffer, the continuity of the playout process is formulated as several constraints of the problem. The buffer level can be interpreted as the urgency of the users, based on which the adaptation could be more reasonable and the radio resource can be utilized more efficiently.

3) Solution of the problem. Due to the scarce and unstable features of the wireless channel, the problem might be infeasible. If the adaptation problem is feasible, the integer programming problem can be solved by a graph model using dynamic programming. Otherwise, a method is given to exclude the users as less as possible to obtain the feasibility.

The remaining of the paper is organized as follows. Section 2.1 introduces the system model. The proposed scheduling scheme is described in detail in Section 3. The simulation results of our algorithm are evaluated and analyzed in Section 4. Finally, a conclusion is drawn in Section 5.

\section{SYSTEM MODEL}

\subsection{System Architecture}

The system architecture is shown in Fig.1. In this paper, we study a normal Long Term Evolution (LTE) wireless system with one eNodeB (Base Station) and several downlink UEs (Mobile Stations). The active users use different categories of UEs to adaptively request the video content stored in the video server. After the video server receives the requests

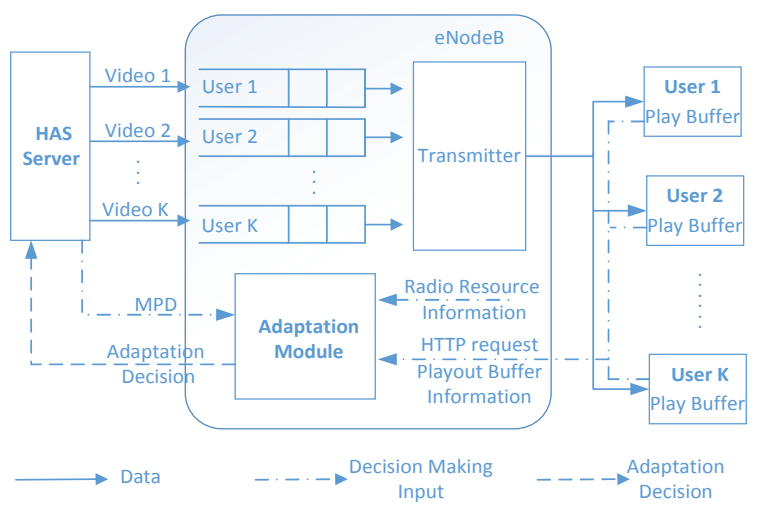

Figure 2: The System Block Diagram

sent by the UEs, the video data will firstly be transmitted to the eNodeB through several wired networks, which are shown as the routers in the figure. Then, according to the resource allocation scheme, the video data will be scheduled and transmitted to the UEs. After all the frames belong to the segment have been fully received, the video segment can be decoded and put into the playout buffer. Finally, the HTTP streaming client can playout the buffered video frames.

The block diagram of the system is shown in Fig.2, which describes the functionality of the adaptation module more specifically. Initially, the server will send the media presentation description (MPD) to the HAS clients so that both the eNodeB and the UEs will get the information of the video segments. Then, the UEs will request for the content according to their interest. For the client-side adaptation schemes, the UEs are responsible for periodically requesting for the segments, while the eNodeB will be responsible for that in our algorithm. The eNodeB will jointly considers the playout buffer level, MPD and the radio resource information to accomplish the adaptation. Here, we assume that the length of the request period equals to the playout duration for a video segment.

As the most promising cellular system, LTE is considered in this paper. Moreover, the extraordinary transmission rate of LTE make it more suitable for providing HTTP streaming services than the other systems. Although LTE is considered specifically here, the algorithm proposed is generic and can be easily extended to some other cellular networks such as Worldwide Interoperability for Microwave Access (WiMAX) and High Speed Downlink Packet Access (HSDPA).

\subsection{QoE Assessing Metric}

The objective of the proposed scheme is to improve the QoE of the video streaming users. Hence, the QoE assessment method could significantly influence the performance of the adaptation. According to the existed works, there are three factors that influence the QoE most.

1) Playout continuity. To eliminate the fluctuation of the wireless channel, the UE is always equipped with a playout buffer, which can store the unplayed video content. If the channel quality degrades, the buffered data can sustain the 
playout process for a while. Nevertheless, when the channel quality doesn't recover from the bad condition, the buffer might be underflow and the playout would be interrupted. According to the analysis in [5], the playout discontinuity could have a severer deterioration to the QoE of the users than the other factors. Frequent or constant interruptions would severely disrupt the users' QoE. To effectively preven$\mathrm{t}$ the interruptions, HAS is designed to match the bit rate of the video and the transmission capability of the wireless channel. Therefore, the playout continuity is regraded as the basic requirements of the users in this paper. By considering the playout buffer level, the resource requirement, which can guarantee the playout continuity, is formulated as a constraint of the problem described in Section 3.

2) Static quality. This metric refers to the quality of a single video frame, which corresponds to the different quality versions. And the higher quality always means the higher bit rate. In this paper, Peak Signal to Noise Ratio (PSNR) is used. It is assumed that there is no packet loss in the network by utilizing the retransmission. Then, the PSNR of each version can be calculated previously in the HAS server, which reduces the requirement of the knowledge of the original video sequence in assessing the QoE. When the eNodeB receives the MPD information, the average PSNR for each segment on each version can be obtained. The PSNR for the $l$-th version of the segment that the $k$-th UE will request is denoted as $P S N R_{k, l}$.

3) Quality smoothness. Although the quality switches can be used to guarantee the continuity, they would deteriorate the QoE if they appear frequently and/or drastically. In [11] and [6], the quality variation is considered as the standard deviation of the quality level of the past several video segments, which is unreasonable. From the perceptual perspective, the more frequent the variations there are, the worse QoE the users will experience even if the static quality is the same. For instance, assume the PSNR of the five consecutive segments are $(33,36,33,36,33)$. Although the PSNR of the first and the fifth segment are the same, the QoE the fifth segment brings is much worse than the first one. Assume there be $S$ switches in the past $T$ seconds and the PSNR variation for each switch is $\triangle P S N R_{s}, 1 \leq s \leq S$. In this paper, the quality variation is considered as a negative influence to the static quality and is denoted as $S * \sum_{s=1}^{S} \Delta P S N R_{s}$. Therefore, the QoE metric for the $k$-th user to request the $l$-th version can be written as in Eq.1, where $a$ is a tunable parameter that is set empirically.

$$
Q o E_{k, l}=P S N R_{k, l}-a * S * \sum_{s=1}^{S} \Delta P S N R_{s}
$$

\section{PROPOSED PLAYOUT BUFFER AWARE ADAPTATION SCHEME OVER MULTI- CLIENT LTE NETWORKS}

\subsection{Problem Formulation}

The parameters to be determined are the adaptation decisions $\tau_{k, l}$ for $k$-th user on the $l$-th version, where the $k$-th user will request for the $l$-th version if $\tau_{k, l}=1$. The objective of the problem is to maximize the total QoE of the users, which has been defined in Eq.1. The total number of the active UEs in the network is $K$ and the total number of the quality versions is $L$. By considering the information of the playout buffer and the radio resource, the problem is formulated as the following integer programming problem.

$$
\begin{array}{ll}
\max & \sum_{k=1}^{K} \sum_{l=1}^{L} Q o E_{k, l} * \tau_{k, l} \\
\text { s.t. } & \text { (1) } T_{k} * N \geq \sum_{l=1}^{L} \sum_{i=1}^{k} \frac{S_{i, l}}{R_{i}} * \tau_{i, l} \\
& \text { (2) } \sum_{l=1}^{L} \tau_{k, l}=1,1 \leq k \leq K \\
& \text { (3) } \tau_{k, l} \in\{0,1\}
\end{array}
$$

The constraints will be explained as follows:

1) The constraint (1) corresponds to the guarantee of the playout continuity. As described above, the playout buffer is mainly for storing the unplayed video content to withstand the fluctuation of the wireless channel. The playout duration of the buffered data in the buffer of the $k$-th UE is denoted as $T_{k}$. To avoid the playout interruption, the requested data should be fully received by the UE in $T_{k}$. More specifically, the transmission rate that the available radio resource can provide should be larger than the requirement of the UE, which is the contraint (1). The left part refers to the available resource. In the LTE system, the smallest resource allocation period is the Transmission Time Interval (1ms). In each TTI, there are $N$ Resource Blocks (RB) can be used and the RBs could be multiplexed in the next TTI. Therefore, the number of the available RBs before the $k$-th UE uses up the buffered data is $T_{k} * N$. Then, the right part corresponds to the requirement of the $k$-th UE, where $S_{k, l}$ is the total size of the $l$-th version of the segment and $R_{k}$ is the average transmission capability of one Resource Block (RB) for the $k$-th UE. $R_{k}$ is calculated as $R_{k}=\frac{T C_{k}}{R B N u m_{k}}$, where $R B N u m_{k}$ is the number of the RBs that have been allocated to the $k$-th $\mathrm{UE}$ and $T C_{k}$ is the total transmission capability provided by the $R B N u m_{k}$ RBs. Therefore, the $\mathrm{RB}$ requirement of the $l$-th version for $\mathrm{UE} k$ is $\left(S_{k, l} / R_{k}\right)$. In addition, the initial condition and the behavior in a special case are discussed. when the algorithm initiates, all the UEs will request for the lowest version of the first segment. Moreover, due to the fluctuation of the wireless channel, the video segment requested in the last request period might cannot arrive on time, which would make $T_{k}$ be 0 . If $T_{k}=0$, the $k$-th UE will continue to wait for the previous requested segment instead of requesting for new segments. So the $T_{k}$ will be set to the length of the requesting period $T_{r e q}$ and the resource requirement $S_{k, l}$ will be set to the residual size of the previous requested segment which can be obtained in the eNodeB.

2) The constraint (2) and (3) are the constraints of the integer adaptation decision variable $\tau_{k, l}$. The constraint (2) indicates that one UE can only request for one version at each adaptation point. And the constraint (3) means the decision for one version can only have two states (1:requested, 0:not requested).

The advantages of the model lie in two aspects. Firstly, compared with the model in [12], the adaptation decisions will be made more reasonably by formulating the constraint of the continuity. For instance, if the buffer level is low, $T_{k}$ is 
small and the UE would request for the low quality version to satisfy the constraint and keep the continuous playout. Otherwise, if the level is high, the constraint can be easily satisfies and the UE can request for the higher versions to maximize the QoE. In fact, the playout buffer level is an important indication of urgency of the clients. The idea here is similar to the one in [9], while it is considered more intelligently in our model. Secondly, the radio resource information is considered, such as the number of the available RBs and the average transmission rate for the UEs, which can effectively prevent the on-off problem mentioned in [8]. Since the UEs are considering jointly in the eNodeB, the UEs would not compete for the shared resource blindly and the resource can be utilized in a rational way to maximize the total QoE.

\subsection{Problem Solving}

Since the wireless channel is unstable, the radio resource might cannot satisfy the requests of all the users, which would make the problem in Eq. 2 infeasible. In this subsection, the feasibility of the problem has to be analyzed firstly. Then, the solving method (if feasible) and the method to obtain the feasibility (if infeasible) are described respectively

From the theoretic perspective, the problem is feasible if the radio resource can meet the requirements when all the UEs request for the lowest quality version. The condition can be formulated in Eq.3. The number of the RBs required to keep the continuous play if the $k$-th UE request for the $l$-th version is denoted as $N(k, l)=S_{k, l} / R_{k}$, where the version 1 is the lowest version. Then, based on the playout duration $T_{k}$, the UEs can be sorted into an ascending order, where UE 1 has the minimum duration $T_{1}$ and UE $k$ has the maximum duration $T_{K}$.

$$
\begin{aligned}
& \text { (1) } N_{1,1} \leq T_{1} * N \\
& \text { (2) } N_{1,1}+N_{2,1} \leq T_{2} * N \\
& \text { ․ } \\
& \text { (K) } \sum_{k=1}^{K} N_{k, 1} \leq T_{K} * N
\end{aligned}
$$

If all the above constraints are satisfied, the problem is feasible. Otherwise, to obtain the feasibility, some UEs have to be chose to not request in the current period. Then, the solution will be discussed in these two conditions.

\section{1) The solution when the problem is feasible}

Based on the constraints (1) in Eq.2, the solution of problem can be converted to finding a path in a graph model. The graph model is noted as $G=(N, E)$, where $N$ is the set of the nodes and $E$ is the set of the edges. In $N$, each node $n_{k, l}$ represents a quality version of a specific user, which has three attributes: the UE index $k_{p}$, the version index $l_{p}$ and the number of RBs required $N_{k, l}$. Given two arbitrary nodes $n_{k_{p}, l_{p}}$ and $n_{l} k_{q}, l_{q}$, if they satisfy

$$
k_{q}=k_{p}+1 \text {, }
$$

there exist a edge $e_{p, q} \in E$ which is direct from $n_{k_{p}, l_{p}}$ to $n_{k_{q}, l_{q}}$. The path $p$ is a direct simple path, which consists of a sequence of nodes $\left(n_{1, l_{1}}, n_{2, l_{2}}, \ldots, n_{K, l_{K}}\right)$ connected by

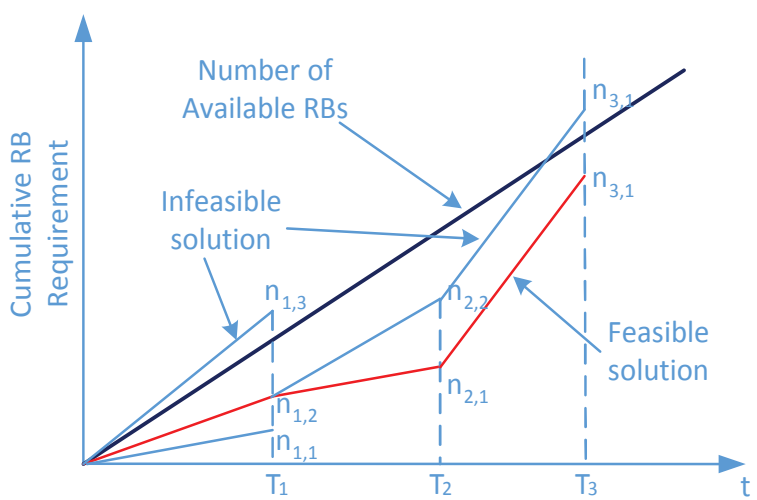

Figure 3: Analysis of the Feasibility

a sequence of $K-1$ edges. Each path $p$ represents an adaptation decision of all the $K$ UEs. Let $n_{1, l_{1}}$ be the start node and $n_{K, l_{K}}$ be the last node. Then only the path that satisfies the constraints in 3 is a feasible one. For a given path, the QoE for each user can be calculated by Eq.1, where the optimal path is the one with the largest total QoE of all the users. The graph model can be explained in Fig.3, where the black bold line refers to the available RBs and the blue line stands for the cumulative requirement of the RBs. A path is feasible if it is always below the the curve of the available RBs, where the path $\left(n_{1,2}, n_{2,1}, n_{3,1}\right)$ plotted as the red line is a feasible one. As we can see, the version 3 of UE 1 is not acceptable and all the paths start from $n_{1,3}$ are not feasible. Meanwhile, any path includes $n_{1,2}$ and $n_{2,2}$ ) is infeasible because even if the lowest version of UE 3 cannot be satisfied.

Based on the graph model, the optimal path can be obtained by the dynamic programming.

2) The method to obtain the feasibility when the problem is infeasible

In this case, some UEs have to be excluded, which mean$\mathrm{s}$ these UEs will not request in the current request period. The UEs will be excluded one at a time until the problem is feasible. The infeasibility is induced by violating the constraints (1) in Eq.2. The satisfiability will be test according to the ascending order of the playout duration $T_{k}$. If the $k$-th constraint is violated, one of the first $k$ UEs should be excluded, which is selected according to the following rules:

- The UEs whose playout duration $T_{k}$ is longer than the length of the request period $T_{r e q}$ will be excluded. Since even if these UEs don't request for new segments, their playout process will not be interrupted and the exclusion of these UEs would deal little damage to the users' QoE. Moreover, these UEs will be reordered according to the value of $T_{k}-T_{r e q}$. The UEs with higher $T_{k}-T_{r e q}$ are less urgent and will be excluded earlier than the other.

- If there isn't any UE with positive $T_{k}-T_{r e q}$, an efficiency metric is designed to decide the sequence of the exclusion. The metric of the $k$-th UE can be written 
Table 1: The configurations of the LTE network

\begin{tabular}{|c|c|}
\hline RB number & 10 \\
\hline Active UE number & 6 \\
\hline Propagation Model & Macro-Cell Urban Mode \\
\hline Turbo Coding Rate & $1 / 2$ or $3 / 4$ \\
\hline eNodeB Power & $\begin{array}{c}43 \mathrm{dbm} \\
\text { uniformly distributed among RBs }\end{array}$ \\
\hline CQI & $\begin{array}{c}\text { Full bandwidth } \\
\text { report period }=1 \mathrm{~ms}\end{array}$ \\
\hline scheduler & Rate Guaranteed (RG) in $[7]$ \\
\hline
\end{tabular}

as

$$
m_{k}=\frac{R_{k}}{S_{k, l} / T_{k}}
$$

The UEs will be excluded according to the ascend order of the efficiency metric, where the UE with the least $m_{k}$ will be the first. The UE with worse channel quality and larger rate requirement will be excluded firstly. In this way, the number of the excluded UEs can be as less as possible to obtain the feasibility of the problem.

When the problem becomes feasible, the solution can be obtained as described in the condition if the problem is feasible.

Last but not least, after the optimal solution is found, the number of the available RBs $T_{r e q} * N$ might be larger than the total requirements $\sum_{k=1}^{K} \sum_{l=1}^{L} S_{k, l} * \tau_{k, l} / R_{k}$. Although the playout continuity of the excluded UEs cannot be guaranteed, the left RBs can be utilized for them to request. The excluded UEs with the highest efficiency metric (defined in Eq.5) will acquire the opportunity and will request for the lowest quality version. This procedure will execute until $\sum_{k=1}^{K} \sum_{l=1}^{L} N_{k, l} \tau_{k, l}>=N * T_{r e q}$

\section{PERFORMANCE EVALUATION}

\subsection{Experiment Setup}

The evaluation is based on a NS3 based LTE simulation platform. Through modifying the application layer in the platform, we embed the real H.264 video encoder and decoder (JVT reference software, JM 16.2) to better simulate the video traffic characteristics. The simulation is based on the network topology as shown in Fig.1 and the details of the LTE networks is shown in Table.1. The scheduler used is the Rate Guaranteed ( $R G$ ) which can guarantee the minimum serving rate of each user. The video sequence used in the test is Foreman that is encoded with the frame rate 30 fps, GOP (Group Of Picture) 12 frames. The total length of the sequence is 3000 frames (50 segments), which is built by duplicating the original sequence (300 frames) 10 times. The original sequence is encoded into five quality versions, of which the bit rate and PSNR for each segment are shown in Table. 2 and Table. 3 respectively.

The compared algorithms involve some client-side schemes that solve the problem in the perspective of a single client(such as Throughput Based scheme (TB) [10] and Buffer
Table 2: Average Bit Rate (kbps) for each segment

\begin{tabular}{|c|c|c|c|c|c|}
\hline Ver Seg & 1 & 2 & 3 & 4 & 5 \\
\hline 1 & 276.772 & 276.652 & 349.024 & 361.864 & 307.148 \\
\hline 2 & 316.912 & 316.412 & 400.604 & 426.148 & 368.648 \\
\hline 3 & 360.828 & 358.128 & 455.016 & 489.436 & 432.208 \\
\hline 4 & 406.768 & 404.12 & 510.948 & 559.816 & 500.908 \\
\hline 5 & 470.664 & 464.868 & 589.392 & 646.668 & 600.804 \\
\hline
\end{tabular}

Table 3: Average PSNR for each segment

\begin{tabular}{|c|c|c|c|c|c|}
\hline Ver Seg & 1 & 2 & 3 & 4 & 5 \\
\hline 1 & 34.7673 & 34.9400 & 35.0512 & 34.3863 & 32.0121 \\
\hline 2 & 35.4151 & 35.6063 & 35.7463 & 35.1485 & 32.8418 \\
\hline 3 & 35.9606 & 36.1530 & 36.3010 & 35.7509 & 33.4494 \\
\hline 4 & 36.5297 & 36.7192 & 36.8643 & 36.4059 & 34.1282 \\
\hline 5 & 37.1930 & 37.3587 & 37.5627 & 37.1145 & 34.9534 \\
\hline
\end{tabular}

Based scheme (BB)[9]), the existing scheme for multi-clients such as Continuum Driven adaptive scheme (CD)[12] and our proposed Playout-Buffer Aware adaptive Scheme over Multi-Client environment (PBMC).

\subsection{Performance of PBMC}

In this subsection, the continuity, static quality and the quality smoothness will be evaluated for the compared algorithms. Firstly, the continuity of the playout process is evaluated. The results of the interruption duration for each algorithm are shown in Fig.4. Based on the playout buffer level, the continuity of the playout process is considered as the constraints in our model, which can effectively avoid or shorten the playout interruption compared with other adaptation schemes. Hence, PBMC acquires the lowest interruption duration as shown in the figure. Since the playout buffer information is ignored by $\mathrm{CD}$, the interruption duration is longer than PBMC. Different from the TB and BB, the adaptation of all the active UEs are considered jointly in $\mathrm{PBMC}$ and $\mathrm{CD}$, which can make the decisions more rationally and reasonably and the on-off problem can be well solved. Therefore, the performance of $\mathrm{BB}$ and $\mathrm{TB}$ are much worse than PBMC and CD. Owing to the consideration of the buffer level, BB behaves better than TB. The reason is that when the buffer level is low, BB will actively degrade the requested level to prevent the buffer underflow, while the requests of $\mathrm{TB}$ are determined only based on the estimated transmission capability.

The average PSNR for all the video segments are shown in Fig.5. Since it's assumed that the six UEs will request at the same time, the on-off problem is likely to occur when using BB and TB. Therefore, the quality of the segments will fluctuate frequently and lots of the radio resource is wasted, which leads to the bad performance of TB and BB. Since $\mathrm{BB}$ will request for the lower quality level to guarantee the playout continuity, the PSNR would be a little lower than TB. As we can see, the knowledge of the available radio resource leads to the increase of the PSNR for PBMC and CD. The PSNR of CD is higher than PBMC because there isn't any constraints of continuity and the UEs could request for higher quality levels. Although CD can acquire better static 


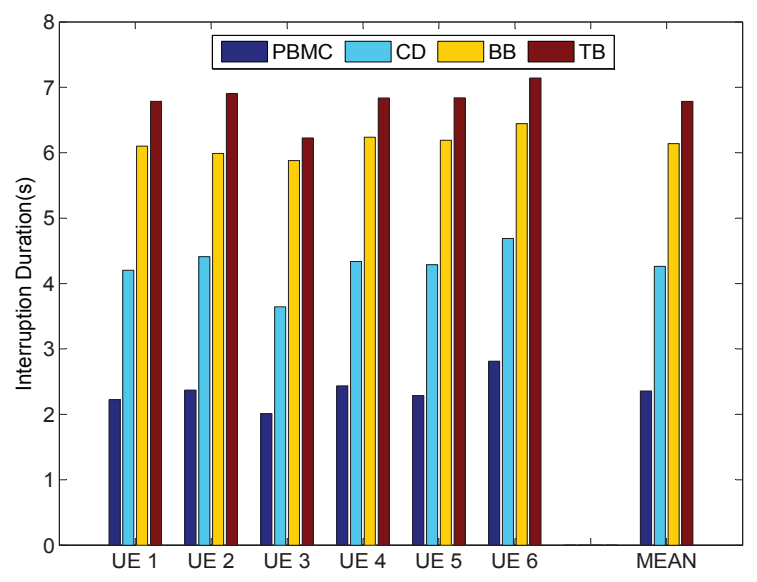

Figure 4: Interruption Duration for the six users under each adaptation scheme

quality, the poor playout continuity makes CD cannot meet the requirement of the HAS over multi-client environmen$\mathrm{t}$, while both the static quality and the continuity can be guaranteed by PBMC.

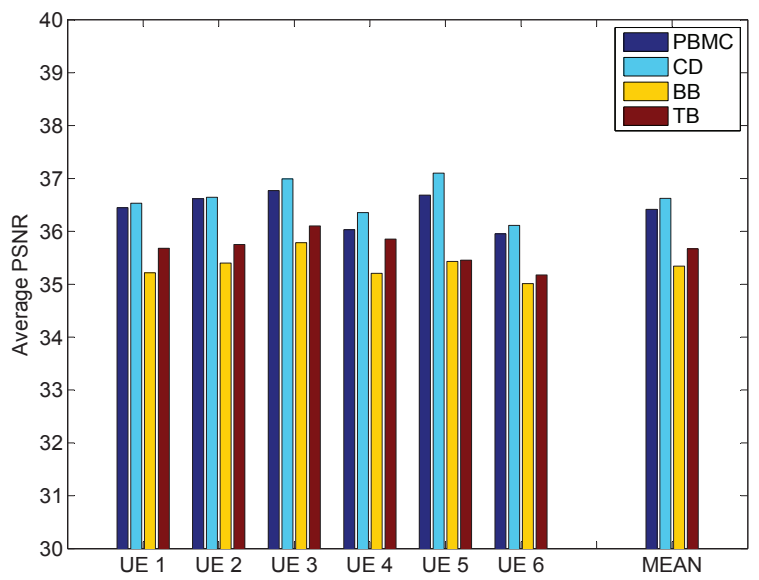

Figure 5: Average PSNR for the six users under each adaptation scheme

Then, the quality smoothness is evaluated. The metric in [12] is employed, which considers the number of quality switches and the intervals between the switches. The quality smoothness metric (QS) is defined as

$$
Q S_{k}=\sqrt{\frac{\sum_{s=1}^{S} n_{s}^{2}}{S}},
$$

where $S$ is the total number of the switches and $n_{s}$ is the number of the segments between the $s$-th and $s+1$-th switches.

As the results in Table.4 show, PBMC and CD can obtain better performance in both the quality smoothness and the fairness by considering the quality smoothness in QoE.

\subsection{Impact of the Available RB}

Table 4: Quality Smoothness for each UE

\begin{tabular}{|c|c|c|c|c|}
\hline QS & PBMC & CD & BB & TB \\
\hline 1 & 4.24 & 3.85 & 2.87 & 2.94 \\
\hline 2 & 3.86 & 4.27 & 2.54 & 3.56 \\
\hline 3 & 4.07 & 3.96 & 5.29 & 3.82 \\
\hline 4 & 4.36 & 3.74 & 3.02 & 3.25 \\
\hline 5 & 4.01 & 4.02 & 5.36 & 5.41 \\
\hline 6 & 3.78 & 3.72 & 1.53 & 2.37 \\
\hline MEAN & 4.05 & 3.93 & 3.44 & 3.56 \\
\hline STD & 0.22 & 0.20 & 1.42 & 1.04 \\
\hline
\end{tabular}

The beahvior of the adaptation scheme largely depends on the transmission capability of the system. Therefore, the performance of the schemes are tested when the number of the available RBs in the system changes. Firstly, Fig.6 depicts the average interruption durations for the six users. With the increase of the available RBs, the interruption duration decreases because the packet delay decreases. When the RB number increases, the playout buffer level will stay at a high level, which makes the decision of $\mathrm{BB}$ mainly depends on the estimated transmission rate that is the same as TB. Hence, the performance of $\mathrm{BB}$ is approaching the one of TB. The main difference between $\mathrm{CD}$ and PBMC is whether to consider the playout buffer level to guarantee the continuity. When the resource is abundant, the high playout buffer level reliefs the weakness of CD, which makes it acquire the performance near to PBMC.

Then, how the average PSNR changes with the number of the available RBs is shown in Fig.7. When the radio resource increases, the PSNR that the user experience increases as well, which means the increasing resource can afford for the transmission of the version with high quality. With the increase of the resource, the performance of TB and BB increase dramatically for the reason that the on-off problem disappears gradually when there is enough resource for all the users to transmit high level versions. From the results shown in Fig.6 and Fig.7, all the schemes can perform well when the radio resource is abundant, while only the PBMC can leads to the least the performance degradation when the radio resource is scarce.

\section{CONCLUSIONS}

In this paper, a playout buffer aware adaptation scheme over multi-client (PBMC) LTE networks was proposed to improve the performance of HAS in the multi-client circumstance. Firstly, a QoE metric was given which considered both the static quality and the quality smoothness. Secondly, to maximize the QoE, an integer programming problem was formulated. By considering the playout buffer level, the resource requirement, which can guarantee the playout continuity, was formulated as a constraint of the problem. Then, the feasibility of the problem was analyzed. If the problem was infeasible, a method was given to exclude the users as less as possible to obtain the feasibility. When the problem was feasible, a graph model can be constructed based on the resource requirement and the playout buffer level. With the model, the solution can be converted to finding a feasible path with the highest QoE in the graph, which can be solved by employing the dynamic programming. Finally, the simulation results can verify the perfor- 


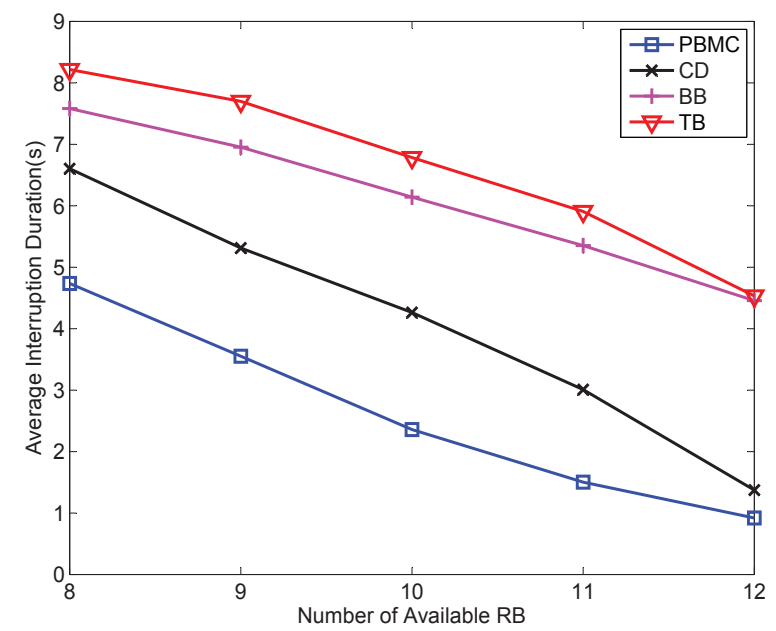

Figure 6: Average Interruption Duration under each adaptation scheme when RB number changes

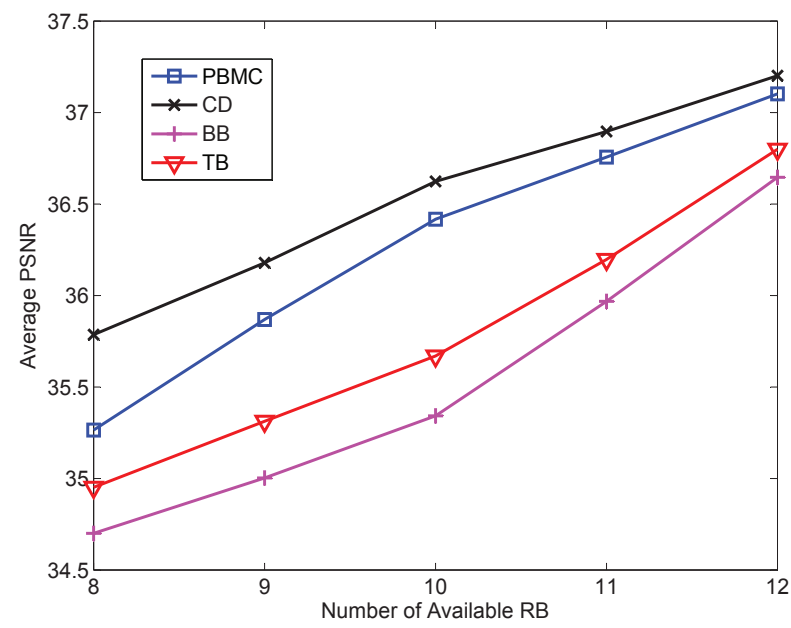

Figure 7: Average PSNR under each adaptation scheme when RB number changes

mance of PBMC in guaranteeing the QoE of all the users in the networks.

The objective of scheduling and adaptation are the same, which is to improve the QoE of the users. Hence, the future work can be considered to improve the performance of HAS by jointly determining the adaptation and the scheduling decisions.

\section{ACKNOWLEDGMENTS}

This work is supported by the National Natural Science Foundation of China Project No.61173110.

\section{REFERENCES}

[1] S. Akhshabi, L. Anantakrishnan, A. C. Begen, and C. Dovrolis. What happens when http adaptive streaming players compete for bandwidth? In Proceedings of the 22nd international workshop on Network and Operating System Support for Digital
Audio and Video, pages 9-14. ACM, 2012.

[2] S. Akhshabi, S. Narayanaswamy, A. C. Begen, and C. Dovrolis. An experimental evaluation of rate-adaptive video players over http. Signal Processing: Image Communication, 27(4):271-287, March 2012.

[3] N. Bouten, S. Latre, J. Famaey, W. Van Leekwijck, and F. De Turck. In-network quality optimization for adaptive video streaming services. Multimedia, IEEE Transactions on, 16(8):2281-2293, November 2014.

[4] Cisco. Cisco visual networking index: Global mobile data traffic forecast update, 2013íc2018. In Cisco Systems Inc ,Feb. 5, 2014.

[5] T. De Pessemier, K. De Moor, W. Joseph, L. De Marez, and L. Martens. Quantifying the influence of rebuffering interruptions on the user's quality of experience during mobile video watching. Broadcasting, IEEE Transactions on, 59(1):47-61, February 2013.

[6] J. De Vriendt, D. De Vleeschauwer, and D. C. Robinson. Qoe model for video delivered over an lte network using http adaptive streaming. Bell Labs Technical Journal, 18(4):45-62, July 2014.

[7] P. A. Hosein. Qos control for wcdma high speed packet data. In Mobile and Wireless Communications Network, 2002. 4th International Workshop on, pages 169-173. IEEE, 2002.

[8] J. C. Jiang, V. Sekar, and H. Zhang. Improving fairness, efficiency, and stability in http-based adaptive video streaming with festive. Networking, IEEE/ACM Transactions on, 22(1):326-340, February 2014.

[9] C. M, S. Lederer, and C. Timmerer. An evaluation of dynamic adaptive streaming over http in vehicular environments. In Proceedings of the 4th Workshop on Mobile Video, pages 37-42. ACM, 2012.

[10] T. C. Thang, Q. D. Ho, J. W. Kang, and A. T. Pham. Adaptive streaming of audiovisual content using mpeg dash. Consumer Electronics, IEEE Transactions on, 58(1):78-85, March 2012.

[11] X. L. Deng, L. Chen, F. Wang, Z. S. Fei, W. Bai, C. Chi, G. L. Han, and L. Wan. A novel strategy to evaluate qoe for video service delivered over http adaptive streaming. In Vehicular Technology Conference (VTC Fall), 2014 IEEE 80th, pages 1-5. IEEE, September 2014.

[12] Z. S. Yan, J. T. Xue, and C. W. Chen. Qoe continuum driven http adaptive streaming over multi-client wireless networks. In Multimedia and Expo (ICME), 2014 IEEE International Conference on, pages 1-6. IEEE, July 2014. 